Emerg Med J 2006;23:304-312. doi: 10.1136/emj.2005.027888

$\mathrm{T}$ his article will consider the identification and management of psychosis, violence, and drug misuse in a pre-hospital and emergency setting.

\title{
Box 1 Article objectives
}
Psychosis:
- Cause
- Identification
- Management
Violence:
- Cause
- Identification
- Management
- Safety
Drug misuse:
- Identification
- Management

R Doy, E J Blowers, E Sutton

\section{PSYCHOTIC ILLNESS}

See end of article for authors' affiliations

Correspondence to:

Rosie Doy, UEA School of Nursing and Midwifery, Edith Cavell Building, Colney Lane Norwich, NR4 TTJ, UK; r.doy@uea.ac.uk
One per cent of the population experience at least one acute episode of schizophrenia ${ }^{1}$ during their life and a similar number will be affected by bipolar disorder (manic depression). These patients have an increased risk of harming themselves-for example, postnatal depression and psychosis is now the leading cause of all maternal deaths by suicide.

Psychosis is therefore a relatively common condition with serious consequences.

\section{FEATURES OF PSYCHOSIS}

Psychosis usually first appears in the late teens or early twenties (slightly later for women). Classically the person is unable to distinguish between reality and thoughts because of hallucinations or delusions.

\section{Box 2 Signs and symptoms of psychosis}

- Delusions-false, fixed beliefs that are not amenable to change despite objective and contradictory evidence to the contrary

- Hallucinations - disordered perception of senses, including hearing voices (auditory), visual, taste (gustatory), smell (olfactory), feeling strange sensations (tactile)-these may cause the person to act on their perceptions

- Mood (affect) disturbances

- Social isolation, withdrawal, and apathy-poor social and living skills, self neglect

- Thought (cognitive) disturbances - the person may believe that thoughts are being inserted into or taken out of their mind; or that their thoughts are being broadcast; they may have disordered thinking and communicate bizarrely or incoherently

- Unpredictable or bizarre behaviour

It is likely that most prehospital and emergency care practitioners will encounter patients in crisis who have an undetected illness (the incidence of new cases of schizophrenia is 1-2 per 10000 population per year), or who are experiencing relapse, or who are deteriorating because of non concordance with medication or lack of engagement with services. 


\section{PRIMARY SURVEY}

Assess the risk of self harm or the potential of harm to others, including yourself. Call for urgent back up if this is the case. You may need to call the police if you think there are real and immediate risks of violence. If you judge management of the patient will require the use of the Mental Health Act, you will need to mobilise an approved social worker (ASW), the patient's GP, or a doctor approved under Section 12 of the Mental Health Act (1983).

Patients who have had a previous episode of psychotic illness are likely to be known to the specialist mental health services, because many are managed completely within primary care.

\section{SECONDARY SURVEY}

The objectives are to obtain an accurate history of the presenting problem, assess the patient's mental state and personality, and to identify possible causes/triggers to the current situation. In addition to the usual history taking (psychiatric, general, and previous medical history), it is useful to assess the appropriateness of the patient's expectations/wishes.

The mental state should then be assessed. This may involve the use of the Mental State or Mini Mental State Examinations-for example, see the Oxford Handbook of Accident and Emergency Medicine (p 580-3). ${ }^{2}$ Another approach to mental state examination is given in Box 14 below.

\section{Box 3 The ABCDE model-initial assessment}

- A Appearance-neglected, dishevelled, inappropriate dress for weather/environment?

- B Behaviour-disinhibited, impulsive, bizarre, aggressive, unpredictable, withdrawn?

- C Communication-incoherent, rapid, and disconnected, confused, limited/uncommunicative?

- D Ideation-beliefs, delusions, preoccupied, perception, hallucinations, thought processes?

- E Emotion-excitable, euphoric, suspicious, sad, inappropriate to situation, rapidly changing (labile)?

\section{DIFFERENTIAL DIAGNOSIS}

It is not always easy to distinguish between medical/ physiological disorders and mental illness presentations. The key is taking a comprehensive history and effective interpersonal and relationship skills. One very important differential is that of a medical condition presenting with disturbance of behaviour of thought (Box 3).

\section{Box 4 Differential diagnoses for psychosis}

Common medical conditions

- $D=$ drugs

- $E=$ endocrine - for example, Cushing's syndrome

- $M=$ metabolic

- $\mathrm{E}$ = ethanol

- $N=$ neoplasia (primary and secondary)

- $\mathrm{T}=$ trauma to head

- 1 = infection (intracranial and systemic)

- $A=$ atherosclerosis and stroke

It is therefore important to screen for features that might indicate an underlying medical problem. History of fits, diabetes, head injury, recent febrile illness, or acute confusion should be excluded. Check the temperature, level of consciousness, and orientation (Box 4). In reviewing these results be aware that the patient's presentation may be complicated by emotional arousal—for example, anger- "self medication" with cannabis (especially in young schizophrenics), and misuse of alcohol by the patient with bipolar disorder or depression.

For further details about the Mental Health Act and relevant sections that may be applied please see Doy et al $(2005)^{3}$ or JRCALC Guidelines (2004). ${ }^{4}$

\section{Box 5 Screening for physical causation}

Medical Assessment/Emergency indicated if:

- Fever

- History of recent fit

- Impaired or depressed level of consciousness

- Known diabetic

- Loss of awareness of surroundings

- Recent onset of confusion, disorientation, and memory impairment

- Signs of head injury/recent history of head injury $(2$ weeks)

- Suspected chest or urinary tract infection

Once a medical cause for the psychosis has been excluded, the patient's history is used to determine what type of psychosis the patient is suffering from (Box 6).

\section{Box 6 Differential diagnoses for psychosis}

Specific mental disorders

- Acute or transient psychotic disorder-usually associated with acute stress and resolves in 2-3 weeks

- Bipolar disorder (also called manic depression)-cyclic disorder characterised by repeated episodes of elevated mood (mania) and depression

- Persistent delusional disorder-characterised by a single delusion or set of related delusions; there are no hallucinations nor disturbances in mood, speech, or behaviour

- Post natal depression and psychosis

- Psychoactive substance abuse

- Schizophrenia

- Severe clinical depression with psychotic features

\section{SCHIZOPHRENIA}

In an acute episode of psychosis, especially the first experience, the person may be absolutely terrified and confused. They may "hear" voices demanding that they harm themselves. ${ }^{5}$ There may be suicidal ideas (about 10\% of patients with schizophrenia will commit suicide within 5 years of the onset of their illness; about $30 \%$ of people with schizophrenia attempt suicide at least once). Male patients, and those who are unemployed, socially isolated, or recently discharged from hospital are most at risk.

\section{BIPOLAR DISORDER}

Bipolar disorder is defined by the National Institute for Clinical Excellence (NICE) as "an episodic, potentially life-long, disabling disorder (with) diagnostic features 


\section{Box 7 Making the diagnosis}

One or two of the following:

- Delusions of being influenced/controlled

- Auditory hallucinations "voices" discussing the patient

- Belief that thoughts are being broadcast, inserted, or removed

- Delusions of superhuman powers, grandiosity

- Persistent hallucinations of any sense

- Irrelevant, incoherent speech, neologisms (new words)

- Marked apathy, poverty of speech

- Incongruent or blunted emotional responses

- Persistent social withdrawal, loss of interest, and aimlessness

- Paranoia and persistent belief that others are seeking to harm self

\section{Box 8 Secondary survey}

Develop rapport and therapeutic relationship taking account of language and culture.

History:

- Check for advanced directives, or a previously documented crisis care plan

- Lifestyle factors-smoking, diet, alcohol, and substance use/misuse

- Medication review, including commencement of atypical antipsychotic medication or increased dosage of antipsychotic/neuroleptic medication

- Concordance with or previous response to treatment/ medication package

- Co-morbidity

Examination:

- Full physical health check-diabetes, hypertension/cardiovascular disease, endocrine disorders, nutritional, and hydration status, chronic obstructive pulmonary disease (60-90\% of people with schizophrenia smoke), medication side effects

Management:

- Involve patients and carers/advocates in care decisions

- Take time to give information to support patients in giving meaningful and valid consent

\section{输 Tip}

Physical examination may also be necessary; schizophrenia is associated with a high mortality, with death on average 10 years earlier than the general population. Cardiovascular disease and/or diabetes are responsible for many of these excess deaths (NICE, 2002'; Prodigy, 2004')

including periods of mania and depression characterised by periods of abnormally elevated mood or irritability, which may alternate with periods of depressed mood. These episodes are distressing and often interfere with occupational or educational functioning, social activities and relationships."

The evidence shows that there is often a considerable delay between the onset of the disorder and first contact with services. Most people experience some changes in mood according to circumstances, but a patient with mania has a persistently high and euphoric mood, which is out of keeping with their circumstances and the environment. In such a situation a hospital admission is often necessary. A key feature of management is to provide a calm, structured environment with avoidance of over stimulation balanced with space for walking to use up excess energy.

\section{Box 9 Signs and symptoms of mania}

- Bizarre or inappropriate behaviour

- Decreased sleep, which may lead to exhaustion

- Delusions and hallucinations

- Elevated mood

- Enhanced libido, flirtatiousness, sexual disinhibition, and promiscuity

- Flight of ideas - racing thoughts

- Grandiose ideas and inflated self esteem

- Impaired concentration, distractibility

- Impaired judgement and decision making

- Impulsive behaviour and recklessness, disregard for danger; spending sprees

- Increased energy and activity levels

- Increased pain threshold

- Irritability, truculence, and aggression

- Labile moods: quickly changing-for example, from elation to anger

- Poor insight

- Pressure of speech, which may be difficult to understand

Hypomania is when patients show symptoms that are not extreme enough to significantly impair work/relationships. It can be managed within primary care.

\section{给 Pitfall}

Many medications can induce the symptoms of mania, including:

- Most antidepressants

- Other psychotropic medication

- Anti-parkinsonian medication

- Cardiovascular and respiratory drugs

- Anti-TB medications

- Gastro-intestinal tract medications

- Steroids

\section{POSTNATAL DEPRESSION AND PSYCHOSIS}

About 70\% of women experience transient "baby blues" 3-5 days after child birth. Other new mothers (10\%) experience postnatal depression that exhibits the same symptoms as a severe (major) depression (see Doy et al, 200533).

This is a serious illness and may present as a crisis situation requiring prompt specialist intervention, including admission of the mother and baby. It is the leading cause of maternal death with four times as many suicides occurring after delivery than in pregnancy itself. ${ }^{7}$ Be aware many women with puerperal psychosis who kill themselves do so later than 6 weeks following delivery of their last child.

The secondary survey in these cases should include the Edinburgh Postnatal Depression Scale. ${ }^{8}$

A small percentage of new mothers $(0.1 \%)$ develop puerperal psychosis-this normally develops within 3 weeks of the birth.

Appropriate in-patient services may be difficult to access depending on locality because there is a national shortfall in the number of mother and baby beds available. However, it is 


\section{Box 10}

Summary of findings related to women who commit suicide:

- $87 \%$ were white women

- $83 \%$ were over the age of 25 years

- $46 \%$ were over the age of 30 years

- $55 \%$ had previous children

- $54 \%$ were seriously ill, either suffering from a postpartum psychosis or a very severe depressive illness

- $50 \%$ had a previous history of serious illness, of whom half had been admitted to a psychiatric unit

- $50 \%$ were in contact with psychiatric services during their index maternity; $75 \%$ of whom were receiving some form of treatment

- Only one woman with significant postpartum mental illness had been admitted to a specialist mother and baby unit

- $65 \%$ of the suicides died violently; half from hanging or jumping from a height. Only $35 \%$ died from an overdose of prescribed medication

These illnesses were therefore neither hidden nor undetected. (Why Mothers Die, 2002). ${ }^{7}$

\section{Box 11 Signs and symptoms of postnatal}

\section{psychosis}

- Delusions and hallucinations (as indicated above)

- Feelings of guilt

- Low mood, which may alternate with elation and euphoria

- Sleep disturbances

- Appetite problems

- Confusion

- Ambivalence towards the baby

your task to ask for urgent assessment by the appropriate mental health team.

\section{RESPONDING TO THE PERSON IN CRISIS}

Whatever the category of psychotic diagnosis, the development of a therapeutic relationship and effective active listening skills are key components in helping the distressed patient in the crisis situation. The patient needs to feel accepted and understood by a practitioner who is trustworthy, interested, helpful, and understanding. Such an approach will help to prevent violence and aggression and facilitate assessment and appropriate referral.

\section{SERVICES AVAILABLE}

In the past, the first occurrence of an acute psychotic crisis or relapse led to admission to an acute in-patient bed. More recently, such patients are managed in the community (Box 13). Crisis resolution and home treatment teams are designed to provide out of hours care for acutely ill patients with psychosis through intensive community home-based support and treatment. This avoids the additional trauma and stigma of a hospital admission. Under the guidance of specialist mental health services rapid tranquillisation may be offered to enhance effective and early intervention and management of the patient.

In summary, effective management of a psychotic crisis requires recognition of the condition, a detailed clinical,
Box 12

Setting:

- Personal and scene safety

- Space to move

Communication:

- Use the patient's name

- Listen to the patient's concerns

- Use appropriate eye contact

- Accept the experienced reality but do not confirm or argue with the patient's beliefs

- Acknowledge the person's feelings-for example, anger, fear

- Explain everything

- Gain consent before talking to the family/carers

Avoid issues that can exacerbate the situation:

- Too many questions

- Too rushed

- Patronising

- Using labels-alcoholic, schizophrenic

- Taking comments personally

Determine:

- Patients' speech (content and style), dress, mannerisms

- Physical signs of illness and/or drug use

- Mismatch between body language and what is said

- Risk assessment and management

Management:

- Aim to manage the current crisis plan and agree clear goals

- Encourage concordance (informed agreement) with taking medication where possible

- Encourage personal control where possible

- Set and state clear boundaries if the person is demanding, disinhibited, and so forth

- Reassure the patient and their family/carers

- Use local referral pathways and liaise with appropriate specialist services

- Very careful reality testing may be helpful-that is, offering alternative explanations

- Maintain detailed documentation

\section{Box 13 Services available (these will vary} according to locality)

- Acute admission units (however there is a national shortage of mother and baby beds)

- Acute day hospitals

- Approved social worker

- Assertive outreach/assertive community teams

- Community mental health teams

- Crisis resolution and home treatment teams

- Duty psychiatrist

- Early interventions' teams

- Mental health link (primary care gateway) workers

- Police

- Primary care teams

- Self help groups

psychiatric and risk assessment, and awareness of the services available (see the National Service Framework for schizophrenia, NICE $2002^{6}$ ).

In the emergency care context it is acknowledged that there will frequently be time pressures, as well as lack of confidence impacting on the practitioner and team's ability to 
fully implement the suggested guidelines and strategies. It is therefore useful for the urgent care worker to establish and develop working relationships with liaison mental health practitioners (in the emergency departments or emergency assessment centres), mental health crisis resolution teams, and mental health link workers (in primary care), so that urgent referrals and joint assessment protocols can be developed.

\section{SUBSTANCE MISUSE}

Following publication of the national drug strategy there has been increased focus on providing seamless treatment and care to substance misusers. ${ }^{9}$ As part of that strategy, treatment tiers and services are identified that are inclusive of all healthcare settings, providing guidance on the levels of intervention, assessment, and expectations of those working within a given tier.

\section{Box 14 Structure of service provision for substance misusers}

Tier 1-non-substance misuse specific services (to include primary care providers; emergency care settings; general medical in-patient settings; general psychiatric care providers)

Tier 2-open access substance misuse services (may include National Health Service and independent sector; health/social care or self referrals accepted; offering advice, brief interventions, support and counselling, low threshold prescribing)

Tier 3-structured community based substance misuse service loffering structured treatment programmes, including substitute prescribing programmes, detoxification, day programmes, and therapies)

Tier 4-residential substance misuse specific services (offering prescribing and rehabilitation)

Highly specialist non-substance misuse specific services

Emergency care settings are acknowledged as being a significant Tier 1 service because of the prevalence of overt or covert substance related presentations within urgent/ unscheduled care. In addition, it is the emergency and primary non-specialist services that commonly offer a starting point for people wishing to engage in a treatment programme.

\section{THE NATURE OF SUBSTANCE MISUSE}

Clinical criteria are available to aid recognition of substance misuse as a health problem, but additionally a number of more holistic and person-centred perspectives are appropriate. Substance misuse may refer to addiction, where physical dependence is implied, but may also include terms such as abuse, dependency, or problematic usage. This varying language can cause confusion in both practitioners and individuals misusing substances leading to potential difficulties in deciding when and indeed if the person is engaging in potentially harmful usage.

If an individual identifies their current use of a substance as causing difficulties in any aspect of wellbeing then it is helpful to accept these concerns as valid and their use should therefore be considered to be problematic. In assessing the presence of substance misuse, a number of themes or criteria are commonly considered and are reflected within both $\mathrm{ICD}^{10}$ and $\mathrm{DSM}^{11}$ criteria.

Box 15 Key diagnostic criteria (not all of which may be present)

Compulsion and cravings: the person describes a subjective awareness of the need or desire to take the substance. This is often strongest during attempts to reduce or stop.

Salience: the substance becomes an increasingly significant part of the person's life, often involving increasing frequency and quantity of substance used. Priority may be given to drug seeking or drug related behaviours relative to other previously valued activities.

Neuroadaption: physical symptoms of dependency may develop, including tolerance and withdrawal.

Use to avoid withdrawal: a pattern of perpetuated or continual use may present due to either physical or psychological "withdrawal". This may include the absence of physical dependency whereby the person has a belief system that without the substance they will be unable to function or cope.

Continued use despite negative effects: this may occur even where the potential damage is quite clear to the individual.

Reinstatement: a rapid or repetitive return to use can be noted after a period of abstinence.

\section{ASSESSING SUBSTANCE MISUSE}

Within "Models of Care", ' guidelines are offered to inform how practitioners working in all tiers of service provision assess. Three levels of assessment are identified, including screening and referral, triage substance misuse assessment, and comprehensive assessment.

Tier 1 services, including emergency departments and unscheduled care settings, are expected to demonstrate consideration of a number of themes as part of a level 1 "screening and referral" assessment. It is expected that all practitioners working within this tier of service are competent in this area.

It is essential that in undertaking an assessment of substance misuse, respect and regard for the patient is demonstrated. Substance misusers often have experienced a number of traumatic life events leading to their use of substances. The reasons why a person may use are complex and multifaceted, ${ }^{12}$ and, as such, moral judgements and assumptions are unhelpful to both the client and practitioner. Although practitioners in urgent and unscheduled care environments may not have the time or opportunity to explore why someone has developed a problem, they can usefully accept that whatever the reason it is valid. A non judgemental, open, and conversational approach to assessment is advocated.

\section{BRIEF INTERVENTIONS}

Individuals presenting with "early" problems can be usefully targeted and brief interventions offered to minimise future significant misuse and maximise long term positive outcomes. ${ }^{13}$ This approach has proved especially beneficial where a person does not present with overt dependence and is particularly relevant to emergency and primary care 
Box 16 Tier 1 Level 1: screening and referral assessment

\section{Content of assessment:}

Identification of substance misuse problem.

Is the person currently using a psychoactive substance? If so, what substance(s), how much, by which method, how often, for how long?

Is usage, stable, increasing?

Is it a problem?

How does the person perceive their use, are they motivated to change?

Identification of related or co-existing problems.

Consider the physical, social, and psychological impact of substance misuse, including medical and psychiatric complications.

Does the person present with co-morbidity? Do they have other health problems unrelated to their substance misuse?

Identification of risks.

What kind of risk may the person face...from the substance and mode of usage, from their own behaviours or physical/mental state, from the social/physical environment they are in?

What types of risk may the person present...to themselves (suicide/self harm), to others including the practitioner, to the physical environment?

Assessment of urgency of referral.

The culmination of previous content gathered. Commonly informed by aspects of risk. Where a risk is identified it may be helpful to consider

Imminence; how soon might the risk event occur?

Severity; how bad will it be if it happens?

Likelihood; how likely do I think it is to happen?

This can be helpful in deciding the urgency of action needed.

Outcome of assessment:

Identification of an appropriate service for referral.

Will be informed by content of assessment, and should consider the person's needs, wishes and motivation.

A non dependent patient may benefit from immediate "brief intervention" (see below).

A low risk patient with relatively uncomplicated presentation and motivation may be advised of or referred to Tier 2 services.

Complex, vulnerable, or high risk presentations where significant need and/or prescribing/detoxification are indicated might be referred directly to Tier 3 services.

practitioners. Brief intervention is extremely effective and may take as little as 15 minutes to complete.

\section{VIOLENCE AND AGGRESSION}

The prevention and management of aggression and violence is a significant challenge for the providers of primary and emergency health care. Within NHS organisations there are 11 incidents per 1000 staff monthly. Incidents within mental health and learning disabilities trusts are reported as 2.5 times higher than the average across all trusts. Ambulance trust staff are also reporting more incidents. Forty three per cent of the incidents reported in acute trusts are within emergency departments. ${ }^{14}$ Healthcare practitioners are at risk from patients, relatives, and the public generally; additionally patients also present risks to each other. ${ }^{15}$
Box 17 The components of brief intervention-

\section{"FLAGS"}

$\mathrm{F}=$ Feedback on risk or health implications because of drug use

$L=$ Listen to the patient's concerns

$A=$ Advise patient about the consequences of continued drug use

$G=$ Goals of treatment should be defined-for example, to reduce or cease drug consumption

$S=$ Strategies for treatment should be discussed-for example, limit setting on use, recognising triggers to use, accessing support. A follow up appointment may be made.

\section{Box 18 Reasons why violence and aggression}

\section{occurs}

- Factors internal to a patient physiological arousal

misperception-for example, of sounds, actions

- psychological aspects-for example, feelings of loss of control, anxiety, fear, acute distress

acute sensory deficits - hearing or sight loss leading to breakdown in communication

unrealistic expectations

- Staff attitudes, lack of understanding of patient expectations

- Organisational factors

- Situational and environmental factors

- Actions of others - for example, family (adapted from NICE, 2005)..$^{16}$

By the very nature of emergency care, individuals who come into contact with services are likely to be experiencing some form of distress. Physical and mental illness (which may include substance misuse and patients suffering from psychosis) can lead to changes in perception, which may increase misinterpretations of the intentions and actions of healthcare staff. Anxiety heightens an individual's senses. Potential cultural difficulties with non verbal communication misunderstandings and misinterpretation as well as language may also trigger distress and frustration. ${ }^{17}$

Anger is not the only prerequisite of violence and aggression, angry people are not always violent, but a link between this powerful emotion and associated potentially dangerous behaviour is accepted. Factors that may exacerbate a person's anger are summarised below.

\section{Box 19 Factors that make people angry}

- Lack of respect

- Not being listened to

- Loss of control

- Sense of injustice

- Discrimination

- Lack of competence in others

\section{PERSONAL AND SCENE SAFETY}

Good risk assessment skills are necessary in the urgent/ unscheduled care arena. Personal and professional safety of practitioners may be largely attributed to good habits and 
systems. It is the good safety behaviour that is carried out all the time that can make the difference in potentially life threatening situations, rather than additional precautions taken in particular circumstances.

Box 20 Summary of patient/environmental risks

- Confusion

- Alcohol or drugs

- History of violence

- Hallucinations and delusions (explored below)

- Longer than normal/expected response time

- Forced entry to gain access to patient

- Crowds-especially at clubs, pubs, or concealed spaces with restricted access

- Lone workers without back up

- Radio black spots

- Domestic violence

- Presence of others perceived as a threat (including police/ security staff)

\section{Tips for enhancing personal safety}

- Good communication systems with agreed codes

- Alarm systems

- Radio provision

- Information links with police about locations

- Flagging systems

- Good quality patient information, including realistic response times

- Lone workers without back up should not attend pub fights or domestic violence situations or locations with known access substance misuse or crowd difficulties (adapted from Department of Health, 2002). ${ }^{18}$

\section{THE ASSAULT CYCLE}

The assault cycle is a theoretical model that offers general advice rather than specific predictions. The trigger phase is a time at which distraction may be important. It is also offers an opportunity to assess and prepare for any potential risks. Escalation is the phase that may lead directly to assault. These phases offer opportunities for skilled de-escalation of a situation. Within the crisis phase a potential assailant experiences physical and psychological arousal. Control over aggressive impulses and the ability for rational thought decreases and this may lead to assault. The practitioner will also experience physical and psychological responses that may influence their control and effectiveness. At this stage self management with a focus on safety issues related to the client, self, and others is important.

After crisis comes the post crisis phase; this is where most intervention errors occur. It is vital to acknowledge the potential for events to "flare up" as significant time is required for individuals to calm both psychologically and physiologically (often cited as at least 90 minutes). In the final recovery phase, an assailant may be mentally and physically exhausted and is commonly remorseful, ashamed, distraught, and despairing. At this stage, individuals may be responsive to interventions designed to relieve guilt that reject the assaultative behaviour but not the individual as a person, understand the incident, and identify strategies to

\section{Box 21 The assault cycle}

- Trigger phase

- Behaviour moves away from baseline-for example, agitation, poor concentration, raised voice

- Interventions

- Identify potential risks

- Early use of de-escalation techniques may include distraction

- Escalation phase

- Behaviour escalates

- Interventions

- Continued use of de-escalation techniques

- Crisis phase

- Patient and staff member are both aroused and assault is imminent or occurring

- Interventions

- Reasoning is no longer possible. Manage own physiological and physical responses with an emphasis on safety of all involved-escape, use of barriers, alarms/shouting to summon help

- Recovery phase

- Patient is calming and returning to baseline

- Interventions

- The most intervention errors occur at this stage be aware of the potential for "flare up" do not attempt an exploration of the incident at this stage

- Post crisis phase

- The patient is low in mood, remorseful, guilty, ashamed, despairing

- Interventions

- Explore the reasons for the incident. Reject the behaviour not the person

- Ensure all others involved receive support

\section{球 Tips-helpful actions}

- Escape (note your escape routes carefully)

- Protecting self by use of barriers

- Engaging the support of others

prevent a recurrence. It is vital that full attention is also given to the needs of the victim.

The assault cycle makes a series of assumptions. These are that violence is often used when someone feels powerless in relation to the professional or the system. Intervention is possible at all times except the crisis phase when physical safety is paramount. The client and the worker experiences high levels of physical or psychological arousal during episodes of aggressive behaviour, and this will affect how they both behave; the practitioner should be able to access appropriate training to develop techniques to overcome this.

\section{DE-ESCALATION}

De-escalation involves the use of techniques to calm down a threatening situation and should be applied early prior to any other interventions being used.

De-escalation involves self awareness of the messages that the worker is conveying through their verbal and non verbal communication. In effective de-escalation, a person shows concern and attentiveness through non verbal and verbal responses, listening carefully, acknowledging any concerns or frustrations, and not being patronising or minimising the 


\section{Box 22 Advice to de-escalate the situation}

- One person should take control and steps must be taken to manage the environment

- Where weapons are involved, a staff member should ask for the weapon to be placed in a neutral location rather than handed over

- Other people may be removed from the area by moving towards a safe place and avoiding being trapped in a corner

- The aggressor may be taken to a quieter but safe area where help is at hand

- Creating space

- Enlisting support from others

- Identify potential risks from others (adapted from NICE 2005). ${ }^{16}$

patient's experience. The worker's non verbal communication is non threatening and not provocative.

\section{Box 23 Key de-escalation principles}

- Attend to non verbal cues, including eye contact

- Allow greater body space than normal

- Adopt a non threatening but safe posture

- Appear calm, self controlled, and confident

- Do not appear dismissive or over bearing

- Explain intentions giving clear, brief, assertive instructions

The worker who has taken control asks for facts about the problem and encourages reasoning by asking open questions and inquiring about the reason for the anger. Threats are avoided. This works to establish rapport and emphasises

\section{Box 24 De-escalation techniques}

- Environmental

- One staff member to take control

- Move to a safe area

- Minimise other distractions such as noise

- Move others

- Create space

- Ensure support is close at hand

- Ask for weapons to be put down

- Consider any sensory impairments

- Attend to the cultural context

- Verbal

- Minimise risks of misinterpretation

- Show concern

- Explain intentions

- Offer and negotiate realistic options

- Avoid threats

- Establish rapport and emphasis co-operation

- Give clear, brief assertive instructions

- Ask open questions

- Listen carefully to concerns; do not minimise these or be patronising

- Non verbal

- Minimise risk for misinterpretation

- Show concern

- Non threatening posture

- Allow personal space

- Manage eye contact

(adapted from NICE 2005). ${ }^{16}$ cooperation offering and negotiating realistic options. Expressions of anger need to be treated with appropriate measured and reasonable responses. In a crisis situation, staff are responsible for taking steps to avoid provocation. It is unrealistic to expect a person exhibiting disturbed/violent behaviour to simply calm down.

\section{POST INCIDENT SUPPORT}

Individuals experience emotional, cognitive, and physiological reactions during the first six weeks after an assault. A single episode of violence or aggression may have a profound impact or the effects may be the result of cumulative abuse. Feelings experienced after an assault include disbelief, helplessness, and frustration, accompanied by resentment and resignation. In most cases these symptoms are resolved after the first six months. It is in this initial most vulnerable period of time when support needs to be available. It is not only the person that is assaulted that suffers; witnesses need support.

Psychological debriefing (also referred to as critical incident stress debriefing) was originally developed for use by groups of emergency workers post incidents. This is a structured, supportive approach led by a trained facilitator.

\section{Box 25 Stages of critical incident stress}

\section{debriefing}

- The introduction of team and establishing ground rules

- Establishing the facts of the incident

- Exploring the thoughts decisions and impressions of those involved

- Exploring emotional reactions

- Normalisation of post trauma reactions offering anticipatory guidance and advice on helpful coping

- Exploring future planning and coping including mobilisation of support

- Disengagement (adapted from Dyregrov 1989). ${ }^{19}$

There is some disagreement as to the effectiveness of this approach, with some suggestion that it may actually be harmful. Individuals have varied coping strategies and it should be made very clear to those who have been involved in incidents that a range of different support is available and that attendance at debriefing is not the only option nor is compulsory.

In summary, the effective recognition, prevention, and management of violence and aggression are of central importance to emergency care staff. This is a complex area encompassing a wide range of reasons why aggression and violence occurs. Recognition and an understanding of the role that staff, patients, and environmental factors play in ameliorating the outcomes in potentially explosive situations is central to the provision of high quality care. Individuals and groups of staff have a vital role to play in maintaining personal and scene safety. This will be promoted by the employment of good safety behaviour all the time with additional precautions as indicated.

\section{Authors' affiliations}

R Doy, E J Blowers, E Sutton, University of East Aglia School of Nursing and Midwifery, Norwich, UK

Competing interests: none declared 


\section{Further reading}

1. Department of Health. Zero tolerance zone fact sheets 2002 www.nhs.uk/zerotolerance/intr.htm laccessed May 2005).

2. NAO. A safer place to work: protecting NHS and ambulance truststaff. London: National Audit Office, 2003.

3. National Institute for Health and Clinical Excellence. The short-term management of disturbed/violent behaviour in in-patient psychiatric settings and emergency departments Clinical Guideline 25. National Institute of Clinical Excellence, 2005.

4. Department of Health. Models of care for substance misuse treatment. London: DH, 2002.

5. UKADU. Tackling drugs to build a better Britain: the Government's 10-year strategy for tackling drug misuse. London: $\mathrm{DH}, 1998$.

6. Mynors-Wallis L, Moore M, Maguire J, et al. Shared care in mental health. Oxford: Oxford University Press, 2002.

7. National Institute for Health and Clinical Excellence, 2002. Schizophrenia: core interventions in the treatment and management of schizophrenia in primary and secondary care. www.nice.org.uk/pdf/CGINICEguideline.pdf (accessed 20 May 2005).

8. Prodigy. Schizophrenia guideline. July 2004. www.prodigy.nhs.uk/guidance. asp?gt = Schizophrenia (accessed 05 May 2005).

9. Simon C, Everitt H, Birtwhistle J, et al. Oxford handbook of general practice. Oxford: Oxford University Press, 2002.

10. Semple D, Smyth R, Burns J, et al. Oxford handbook of psychiatry. Oxford: Oxford University Press, 2005.

11. National Institute for Health and Clinical Excellence. Bipolar disorder clinical guidelines scope. www.nice.org.uk/ Biploar_Scope_FINAL.pdf (accessed 15 May 2005).

12. Doy R, Burroughs $D$, Scott J. ABC of community emergency care-issues in mental health-consent, the law and depression-management in emergency settings. EMJ 2005;22(4):279-85.

13. Joint Royal Colleges Ambulance Liaison Committee, 2004. Clinical Practice Guidelines version 3.0. University of Warwick/JRCALC. www.nelh.nhs.uk/ emergency (accessed 15 May 2005).

\section{REFERENCES}

1 Prodigy. Schizophrenia guideline. July 2004. www.prodigy.nhs.uk/ guidance.asp?gt = Schizophrenia (accessed 8 May 2005).

2 Semple D, Smyth R, Burns J, et al. Oxford handbook of psychiatry. Oxford: Oxford University Press, 2005.

3 Doy R, Burroughs D, Scott J. ABC of community emergency care-issues in mental health-consent, the law and depression-management in emergency settings. EMJ 2005;22(4):279-85.

4 Joint Royal Colleges Ambulance Liaison Committee (JRCALC). Clinical practice guidelines version 3. University of Warwick/NHS, June, 2004.

5 Andrews G, Jenkins R. Management of mental disorders volume 2. London: World Health Organisation (UK Edition), 1999.

6 National Institute of Clinical Excellence. Schizophrenia core interventions in the treatment and management of schizophrenia in primary and secondary care. London: NICE, 2002.

7 Why Mothers Die 2000-2002 - Report on confidential enquiries into maternal deaths in the United Kingdom, RCOG/CEMCH. www.cemach.org.uk/publications/WMD2000 2002/ content.htm (accessed 16 September 2005).

8 Cox J, Holden J, Sagovsky R. Detection of postnatal depression: development of the 10-item Edinburgh Postnatal Depression Scale. B J Psychiatry 150:782-876.

9 UKADU. Tackling drugs to build a better Britain: the Government's 10-year strategy for tackling drug misuse. London: DH, 1998.

10 World Health Organisation, International Classification of Diseases (ICD 10). Classification of mental and behavioural disorders. Geneva: WHO, 1992.

11 American Psychiatric Association. Diagnostic and statistical manual of mental disorders. DSM IV-TR. Washington, American Psychiatric Association. 2000.

12 Peterson T. 'Exploring substance misuse and dependence: explanations, theories and models'. In: Peterson T, McBride A, eds. Working with substance misusers, a guide to theory and practice. London: Routledge, 2002

13 Hulse G, White J, Conigrave K. 'Identifying treatment options'. In: Hulse G White J, Cape G, eds. Management of alcohol and drug problems. Oxford: Oxford University Press, 2002.

14 NAO. A safer place to work: Protecting NHS and ambulance trust staff. London: National Audit Office, 2003.

15 Chambers N. 'We have to put up with it-don't we?' The experience of being the registered nurse on duty, managing a violent incident involving an elderly patient; a phenomethodological study. J Adv Nurs 1998;27:429-36.

16 NICE. The short-term management of disturbed/violent behaviour in in-patient psychiatric settings and emergency departments. Clinical Guideline 25. National Institute of Clinical Excellence, 2005.

17 Paterson B, Leadbetter D, McComish A. De-escalation in the management of aggression and violence. Nurs Times 3 Sept 1997:93(36):58-61.

18 Department of Health. Zero Tolerance Zone Fact Sheets, 2002. www.nhs.uk/ zerotolerance/intr.htm (accessed May 2005).

19 Wright S, Gray RK, Parkes J, et al. Therapeutic management of violence a literature review, 2002. www.nmc.org.uk (accessed May 2005). 